\title{
Visualization of oxidative stress in ex vivo biopsies using electron paramagnetic resonance imaging
}

Håkan Gustafsson, Martin Hallbeck, Mikael Lindgren, Natallia Kolbun, Maria Jonson, Maria Engström, Ebo de Muinck and Helene Zachrisson

\section{Linköping University Post Print}

\section{Tweet}

N.B.: When citing this work, cite the original article.

Original Publication:

Håkan Gustafsson, Martin Hallbeck, Mikael Lindgren, Natallia Kolbun, Maria Jonson, Maria Engström, Ebo de Muinck and Helene Zachrisson, Visualization of oxidative stress in ex vivo biopsies using electron paramagnetic resonance imaging, 2015, Magnetic Resonance in Medicine, (73), 4, 1682-1691.

http://dx.doi.org/10.1002/mrm.25267

Copyright: Wiley

http://eu.wiley.com/WileyCDA/

Postprint available at: Linköping University Electronic Press

http://urn.kb.se/resolve?urn=urn:nbn:se:liu:diva-113407 
Visualisation of oxidative stress in ex vivo biopsies using electron paramagnetic resonance imaging (EPRI)

H. Gustafsson ${ }^{1,2, ~}{ }^{*}$, M. Hallbeck ${ }^{3}$, M. Lindgren ${ }^{4,5}$, N. Kolbun ${ }^{1,2}$, M. Jonson ${ }^{5}$, M. Engström ${ }^{1,2}$, E. de Muinck $^{6}$, H. Zachrisson ${ }^{6}$

1. Department of Medical Technology (MTÖ), Radiation Physics, Department of Medicine and Health Sciences, Linköping University, Linköping, Sweden

2. Center for Medical Image Science and Visualization (CMIV), Linköping University, Sweden.

3. Department of Clinical Pathology and Clinical Genetics, and Department of Clinical and Experimental Medicine, Linköping University, Linköping, Sweden.

4. Department of Physics, Norwegian University of Science and Technology, Trondheim, Norway.

5. IFM-Department of Chemistry, Linköping University, Linköping, Sweden.

6. Department of Medical and Health Sciences (IMH), Division of Cardiovascular Medicine, Linköping University, Sweden.

* Corresponding author.

Contact details:

E-mail: hakan.l.gustafsson@liu.se

Address: Håkan Gustafsson, IMH/Radiation Physics, Linköping University, 58185 Linköping, Sweden

Telephone: +46 (0)101043023 or +46 (0)709 428446

Fax: Not available

Word count: 4314 
ABSTRACT

\section{Purpose}

The purpose of this study was to develop an X-Band electron paramagnetic resonance imaging (EPRI) protocol for visualisation of oxidative stress in biopsies.

\section{Methods}

The developed EPRI protocol was based on spin trapping with the cyclic hydroxylamine spin probe 1hydroxy-3-methoxycarbonyl-2,2,5,5-tetramethylpyrrolidine (CMH) and X-Band EPR imaging. Computer software was developed for deconvolution and back-projection of the EPR image. A phantom containing radicals of known spatial characteristic was used for evaluation of the developed protocol. As a demonstration of the technique EPRI of oxidative stress was performed in six sections of atherosclerotic plaques. Histopathological analyses were performed on adjoining sections.

\section{Results}

The developed computer software for deconvolution and back-projection of the EPR images could accurately reproduce the shape of a phantom of known spatial distribution of radicals. The developed protocol could successfully be used to image oxidative stress in six sections of the three ex vivo atherosclerotic plaques.

\section{Conclusion}

We have shown that oxidative stress can be imaged using a combination of spin trapping with the cyclic hydroxylamine spin probe $\mathrm{CMH}$ and $\mathrm{X}$-Band EPR imaging. A thorough and systematic evaluation on different types of biopsies must be performed in the future to validate the proposed technique.

\section{Key Words: EPR, EPR imaging, EPRI, spin trap, oxidative stress, reactive oxygen species}




\section{INTRODUCTION}

Overproduction of reactive oxygen species (ROS) such as superoxide radicals $\left(\mathrm{O}_{2}{ }^{-}\right)$and hydroxyl radicals $(\mathrm{OH} \bullet)$ can lead to oxidative stress with subsequent oxidative damage to tissues. ROS are also known to be important for physiological functions such as for elimination of pathogens and as cell signalling molecules (1-2). It is well-known that ROS are involved in the pathology of a large number of diseases (3) and it has been shown that oxidative stress is a key factor in the initiation and progression of atherosclerosis (4) in the development of endothelial dysfunction (5) and hypertension (6).

Measurement of radicals in human tissues is a methodological challenge because of high reactivity and short half-life of reactive oxygen and nitrogen species which leads to difficulties for direct determination. Electron paramagnetic resonance imaging (EPRI) is a technique for imaging of paramagnetic species (7) and provides a unique possibility to image the distribution of free radicals in medical samples. EPRI is being developed towards in vivo monitoring and imaging of radicals in mice (8-10) and initial attempts of in vivo EPR in humans have been conducted (11-12).

EPRI has several similarities with (nuclear) magnetic resonance imaging (MRI), but the progress of EPRI has been slow compared to MRI. This is partly due to several technical difficulties associated with the often very low concentrations of paramagnetic species in the samples of interest and the fast electronic relaxation times. (T1 is typically in the order of milliseconds to seconds for protons as compared to 1 to 20 microseconds for electrons (13)). The development of pulsed EPRI methods has therefore been hindered and EPRI is therefore typically performed using filtered back-projection of a set of repeated continuous wave (c. w.) experiments for different combination of gradient angles (1415). However, recent progress of EPR imaging is promising and may open for a large number of important clinically applications. Nevertheless, visualisation of oxidative stress in biopsies and in vivo needs to be further developed.

Visualisation of radicals using EPRI is based on the local interaction between the tissue and molecular probes (EPR spin traps/probes)(16-17). Two main techniques can be used: (1) The rate constant (halflife) of the reduction of a nitroxide free radical to an EPR silent diamagnetic hydroxylamine has been observed in comparisons of images obtained at different time after the incubation/injection with the nitroxide free radical. The rate constant of the reduction of the nitroxide free radical is dependent on the tissue redox status and the time dependent loss of signal in the images can be used to assess tissue redox status. Rapid loss of signal as compared to normal tissue thus indicates that the tissue is highly 
reducing compared to normal tissue. There are examples in literature such as in the incubation with TEMPO (2,6,6-Tetramethylpiperidine-1-oxyl) for studies of UV-induced ROS in human skin biopsies (18)) and injection of 3-CP (3-carbamoyl-2,2,5,5-tetramethylpyrrolidine-N-oxyl) in mice for imaging of tumour redox status (19)). Another method (2) for imaging of ROS is the incubation or injection of an EPR silent diamagnetic cyclic hydroxylamine. The diamagnetic cyclic hydroxylamine will react with ROS and accumulate as a free radical in regions containing high concentrations of ROS. This will be observed as regions with high signal intensity. While cyclic hydroxylamines (20-21) has been shown to be especially useful for detection of ROS in cardiovascular studies (22), to the best of our knowledge no one has used the combination of EPR imaging with EPR silent diamagnetic cyclic hydroxylamines for imaging of oxidative stress with high signal intensity in regions with oxidative stress as we demonstrate here.

The diamagnetic cyclic hydroxylamine spin probe 1-hydroxy-3-methoxycarbonyl-2,2,5,5tetramethylpyrrolidine $(\mathrm{CMH})$ is known to react with superoxide, peroxyl radical, nitrogen dioxide and peroxynitrite (but do not react with $\mathrm{H}_{2} \mathrm{O}_{2}$ or nitric oxide) $(20,23-24)$. The oxidation of $\mathrm{CMH}$ leads to the formation of the paramagnetic 3-methoxycarbonyl-proxyl nitroxide (CM•) (24) and it is known that a majority of the formed $\mathrm{CM} \bullet$ in vitro and in vivo is due to oxidation of $\mathrm{CMH}$ by superoxide (25-26).

Vulnerable atherosclerotic plaques are prone to rupture and frequently lead to stroke or myocardial infarction. Vulnerable plaques are characterized by an increased number of inflammatory cells such as macrophages and related inflammation, especially in the cap, might lead to cap rupture as well as thrombus formation. High local concentrations of immune cells such as macrophages are correlated to regions of high oxidative stress (27) and it has been hypothesized that this leads to increased activity of matrix metalloproteinase which degrades the fibrous cap and therefore further destabilise the cap of atherosclerotic plaques with subsequent risk of cap rupture (28).

The aim of this work was to develop a new method and an imaging protocol for imaging of ROS in biopsies. The method was based on incubation with an EPR silent diamagnetic cyclic hydroxylamine and observing high signal intensity in regions with possible high oxidative stress using X Band EPR imaging. The developed protocol was demonstrated for imaging of ROS in six sections of three symptomatic ex vivo atherosclerotic plaques (two sections from each plaque).

\section{METHODS}

\section{EPRI phantom}


An EPRI phantom was made using $1.82 \mathrm{~g}$ gelatine leafs (gelatine from animal origin, Dr. Oetker, Mölndal, Sweden), which were dissolved in $0.35 \mathrm{dl}$ water with $1 \mathrm{mM}$ TEMPO (2,2,6,6-Tetramethyl1-piperidinyloxy) (Aldrich 214000-5G) in a beaker using a magnetic stirrer/heater until completely dissolved. An EPR tissue cell (WG-806-A-Q-P Suprasil tissue cell, Wilmad-LabGlas, Vineland, New Jersey, USA) was completely filled with the gelatine/TEMPO mixture and the gelatine was allowed to solidify completely at $4{ }^{\circ} \mathrm{C}$. The gelatine with TEMPO could thereafter be cut into desired shapes and sizes. A $6 \mathrm{~mm}$ diameter circle was used as the EPRI phantom to be reproduced in the imaging reconstruction software as discussed below.

\section{Patient data and duplex ultrasound in vivo}

Three patients (a woman 64 years old, a man 74 years old and a man 85 years old) undergoing carotid endarterectomy (CEA) was prospectively enrolled for study participation. The study was approved by the Local Ethical Review Board in Linköping, Sweden. The participants were both written and verbally informed about the purpose of the study and provided their written informed consent to participate. All patients had symptomatic internal carotid artery (ICA) stenosis [minor stroke $\mathrm{n}=2$; transient ischemic attack (TIA), $\mathrm{n}=1]$. Preoperative duplex ultrasound of the neck vessels was performed within one week before carotid endarterectomy at an angle of 60 degrees at maximum between the transducer and the blood flow direction using a high frequency ultrasound Acuson S2000 scanner (Siemens, Mountain View, USA) equipped with a $9 \mathrm{MHz}-18 \mathrm{MHz}$ transducer. Ultrasound images/data were saved for further offline analysis using computerized image analysis software; Adobe Photoshop CS5 (Adobe systems, Mountain View, USA). The degree of internal carotid artery stenosis was measured as an evaluation of the peak systolic flow velocity (PSV), i.e. the European Carotid Surgery Trial (ECST) method (29). Plaque composition and cap surface were objectively assigned.

\section{Carotid endarterectomy, plaque transport and storage}

Immediately after carotid endarterectomy the plaque was placed in a small plastic bottle and snapfrozen in liquid nitrogen. The plaque was transported to the EPRI laboratory and stored for up to 19 days in liquid nitrogen until the time of cryosection and subsequent EPRI.

\section{Spin trap solution}

$0.01 \mathrm{M}, \mathrm{pH}=7.4$ phosphate buffered saline (PBS) (Sigma P3813-10-PAK) with $100 \mu \mathrm{M}$ diethylene triamine pentaacetic acid (DTPA) (Sigma-Aldrich D6518-5G) was kept on ice and bubbled with nitrogen gas for approximately 30 minutes prior to addition of CMH (1-hydroxy-3-methoxycarbonyl 2,2,5,5-tetramethylpyrrolidine) (Noxygen Science Transfer \& Diagnostics GmbH. NOX-02.1-50mg) to a final concentration of $30 \mathrm{mM} \mathrm{CMH}$. This spin trap solution was kept on ice and under constant 
bubbling with nitrogen gas until incubation of carotid plaque slices (see section below) to ensure an oxygen free environment.

\section{Cryosection}

The plaques were equilibrated to $-20^{\circ} \mathrm{C}$ for 90 minutes in a MICROM HM 550 Cryostat (MICROM International GmbH, Walldorf, Germany) before mounted to a sample plate using Tissue-Tek O.C.T compound (Histolab, Göteborg, Sweden). Cryosectioning was performed at $-20^{\circ} \mathrm{C}$ chamber temperature, with a knife temperature of $-17^{\circ} \mathrm{C}$. Histology slices for Hematoxylin and Eosine staining (H\&E) were cut with a thickness of $20 \mu \mathrm{m}$, while slices for EPRI had a thickness of $250 \mu \mathrm{m}$. The slices for H\&E staining were placed on SuperFrost Plus slides ( Menzel-Gläser, Braunschweig, Germany), dried at room temperature for 30 minutes and stored at $-20^{\circ} \mathrm{C}$ until use. Slices for EPRI were placed on an EPRI tissue cell and immediately transported to the EPRI laboratory.

\section{Incubation with spin trap and tissue cell.}

Immediately after cryosection the $250 \mu \mathrm{m}$ slices for EPRI were placed on the EPRI tissue cell and transported to the EPRI laboratory. Time for transport was approximately 2 minutes, which allowed the samples to thaw slightly. Incubation was performed using $30 \mu \mathrm{L}$ of the spin trap solution (see section above) during exactly three minutes and thereafter all surplus of the solution was gently removed using a piece of paper on the sides of the plaques. Incubated plaques were thereafter covered using Parafilm (Pechiney Plastic Packaging Company, Chicago, IL) to avoid drying and deterioration of the biopsy.

\section{EPRI}

EPRI was performed using an X Band $(9.6 \mathrm{GHz})$ Bruker E540 EPR and EPR imaging spectrometer (Bruker BioSpin GmbH, Rheinstetten, Germany) equipped with a E540 GC2X two axis X band gradient coil set with gradients along y axis (along sample tube) and $\mathrm{z}$ axis (along $\mathrm{B}_{0}$ ). An ER 4108TMHS resonator was used suitable for the $25 \mathrm{~mm}$ air gap between the gradient coils. All measurements were performed in room temperature. During measurements, the samples were placed on the WG-806-A-Q-P Suprasil tissue cell. An EPR spectrum obtained without gradients was obtained for the deconvolution (see section below) using the following parameters: applied microwave power $10.02 \mathrm{~mW}$, modulation amplitude $0.150 \mathrm{mT}$, modulation frequency $100.0 \mathrm{kHz}$, sweep width $15.94 \mathrm{mT}$, points 512, sweep time: $10.01 \mathrm{~s}$, time constant $10.24 \mathrm{~ms}$, number of accumulated scans 5. EPRI was performed using the same parameters as for EPR spectroscopy with the addition of the following imaging specific parameters: field of view (FOV): $14.00 \mathrm{~mm} * 14.00$ $\mathrm{mm}$, gradient strength $(\mathrm{G}) 3.000 \mathrm{mT} / \mathrm{cm}$, pixel size $0.500 \mathrm{~mm} * 0.500 \mathrm{~mm}$, first gradient angle $4.286^{\circ}$, number of gradient angles 21 , spectrum width $5.000 \mathrm{mT}$. The paramagnetic 3- 
methoxycarbonyl-proxyl nitroxide $(\mathrm{CM} \bullet)$ is very stable compared to the measurement time used in the present study (22) and the total measurement time was therefore of less importance. However, there is a constant, but slow, increase of $\mathrm{CM} \bullet$ in regions with e.g. superoxide and the acquisition time was therefore limited to 25 minutes to keep an approximately constant signal-to-noise ratio during acquisition.

\section{EPRI image reconstruction}

A standard personal computer (PC) (2.4 GHz processor, $4 \mathrm{~GB}$ of RAM, Windows 7$)$ was used as a computing platform to run the developed Matlab (Matlab 7.1 Release 2010a) code for image reconstruction. The developed Matlab code is given in the supplementary information and requires installation of the Easyspin toolbox (30) and has similarities of a deconvolution method previously published (31). Each acquired projection (see section above) was deconvolved with the EPR spectrum obtained without gradients using deconvolution with a Gaussian filter (see line 65 in the code in the supplementary information). Full-width-at-half-maximum (FWHM) of the Gaussian filter was 100, 300 or 500 points out of 1024 points in the frequency domain (see line 59-68 in the code given in the supplementary information). Image reconstruction was performed using the deconvoluted projections by means of filtered back projection using the standard iradon Matlab routine with Shep-Loggan filter of $0.1,0.2$ or 0.3 (a scalar between 0 and 1 that rescales the frequency axis of the filter) for noise reduction (see line 96 in the code given in the supplementary information). For the parameters given in the section above this method for signal deconvolution and filtered back projection resulted in an image with $362 * 362$ image pixels with $0.1465 \mathrm{~mm} /$ pixel. Millimetre scale in the reconstructed images was calculated as $10 *(\mathrm{~B} 0-\mathrm{Bc}) / \mathrm{G}$ where $\mathrm{B} 0$ is the magnetic field vector, $\mathrm{Bc}$ is the centre field and $\mathrm{G}$ is the gradient strength in $\mathrm{G} / \mathrm{cm}$ (see lines $85-93$ in the supplementary information).

\section{Histopathology}

Next to the sections used for EPRI $20 \mu \mathrm{m}$ thick frozen slices were taken for histopathological analysis. These sections were fixed in $100 \%$ alcohol, stained with standard Hematoxylin and Eosine staining (H\&E) and subsequently digitalized (ScanScope AT Turbo, Aperio). Analysis were done of the digitalized sections using ImageScope (Aperio) regarding cap thickness, presence of cap rupture and the presence of foamy macrophages within the cap. The digitalized image were subsequently orientated and compared to the images produced by EPRI.

\section{RESULTS}

\section{EPRI of reference phantoms}


The imaging protocol was calibrated and evaluated using a phantom of known size and shape. This was particularly important to be able to obtain precise geometrical parameters of the measured heterogeneous radical distribution in unknown biological samples. Thus, a phantom (Figure 2, Panel A) was constructed from gelatine containing $1 \mathrm{mM}$ TEMPO to verify the image reconstruction using a well-defined sample with high enough signal-to-noise ratio. While the EPRI spectrometer used for this work allows gradient strengths up to $17 \mathrm{mT} / \mathrm{cm}$, the imaging experiments described here were performed using a modest gradient strength $(\mathrm{G})$ of only $3 \mathrm{mT} / \mathrm{cm}$. The reason for this was that preliminary experiments had showed that $3 \mathrm{mT} / \mathrm{cm}$ was optimal for the limited signal intensity available from incubated sections of atherosclerotic plaques. Even though higher gradient strengths give the theoretical possibility of increased image resolution, it is necessary to have high enough signal-to-noise ratio to be able to perform a successful deconvolution process.

For this sample EPR spectra after deconvolution as a function of gradient angle in the ZY plane are shown in Figure 3. The left panel is a 2D plot showing the EPR spectra after deconvolution as a function of gradient angle, color-coded so that yellow to red is more positive amplitude and green to blue is less amplitude. Representative EPR spectra after deconvolution for two particular gradient angles $\left(\theta=4^{\circ}\right.$ and $\left.\theta=90^{\circ}\right)$ are shown in the right panel.

The developed software (described in the experimental section above and in the supplementary information) was tested for image reconstruction of phantoms with radical distributions of known sizes and shapes. As an example we show the analysis of the phantom shown in Figure 2A: We studied the impact of the filter parameter used in the Gaussian deconvolution and the Shepp-Logan filter parameter used in the filtered back-projection on the signal-to-noise ratio and resolution along the horizontal axis of the phantom shown in Figure 2. In Figure 4, Panel A, the EPRI signal intensity is showed for one line along the horizontal axis in the centre of the circular phantom for different settings of the Gaussian filter used in the deconvolution (for an constant Shepp-Logan filter parameter in the back-projection). A Gaussian filter with 100 points gave high signal-to-noise ratio but limited resolution (red curve in Figure 4A). Resolution was increased with increased number of points in the Gaussian filter (blue and black curves in Figure 4, Panel A), however, at the expense of lower signalto-noise ratio. We conclude by trial and errors that 300 points in the Gaussian filter for deconvolution is a good compromise between resolution and signal-to-noise ratio. Similarly; the Shepp-Logan filter used in the filtered back-projection (section 2.8), was optimised by trial and error (Figure 4B) and it was found that a filter of 0.3 was optimal for best resolution without compromising the signal to noise ratio. As can be read from the z-axis, in all cases of filter testing, the radical distribution is well contained within the $6 \mathrm{~mm}$ diameter of the phantom. Using the optimized filter parameters for deconvolution and filtered back-projection, reconstruction the EPRI of the gelatine phantom could be 
calculated as shown in Figure 2 (Figure 2, Panel B) (image reconstruction using fwhm $=300$ points for the Gaussian filter in the deconvolution and a Shepp-Logan filter of 0.3 in the filtered backprojection). Figure 2, Panel C shows a semi-transparent EPRI-image overlaid on the photograph of the phantom. As can been seen in Figure 2, Panel A the shape of the phantom was no perfectly cylindrical and a close examination also revealed that the thickness of the gelatine/TEMPO was not perfectly uniform. However, there was a good agreement between the distribution of free radicals and the shape and size of the phantom.

\section{Patient data and duplex ultrasound in vivo}

In vivo duplex ultrasound showed that the plaque composition was atheromatous in one plaque and atheromatous with extensive calcification in two cases. The cap was classified as extensively ulcerated in all three plaques. The degrees of the internal carotid artery (ICA) stenosis measured as peak systolic flow velocities (PSV) were $5.3 \mathrm{~m} / \mathrm{s}, 7.0 \mathrm{~m} / \mathrm{s}$ and $3.0 \mathrm{~m} / \mathrm{s}$ respectively indicating high grade stenosis $80 \%$ - $99 \%$ on the ipsilateral (symptomatic) side (ECST (32)).

\section{EPRI of atherosclerotic plaques}

Having verified the image reconstruction algorithm, EPRI was performed for totally six sections $(\mathrm{N}=6)$ from the three atherosclerotic plaques (two separated sections from each of the three patients). A typical example is shown in Figure 5 and 6 (plaque 1, section 1) where the deconvoluted EPR spectra as a function of gradient angle are shown in Figure 5 (left panel) and the associated deconvoluted EPR spectra for two particular gradient angles $\left(\theta=4^{\circ}\right.$ and $\left.\theta=90^{\circ}\right)$ are shown in Figure 5 (right panel). As shown, the EPR signal was relatively strong with good signal to noise ratio and there were no problems to reconstruct the free-radical concentration map from these raw data.

Figure 6 shows a photograph of the section (plaque 1, section 1) located on the EPR tissue cell immediately after cryosection, incubation with spin trap and after covering with Parafilm (Panel A). Panel B shows the EPRI image obtained after reconstruction of the data shown in Figure 5. Note that most part of the plaque gives some EPRI intensity but certain areas shows high concentration of spin probe signal. Panel C shows the areas with highest EPRI intensity fused onto the photograph. Panel D shows the immunohistochemistry of an adjacent slice of which the slice where the EPRI was carried out. It is emphasized that this is not the identical sample and that the section is somewhat displaced in relation with the section used for EPRI, that was mounted on an EPR tissue cell. Despite these small discrepancies one can easily identify the important regions and carry out immunohistochemistry to be further discussed below. 
Figure 7 is similar to Figure 6 but from a different section from a different atherosclerotic plaque (plaque 2, section 1). Panel A shows a photograph of the section located on the EPR tissue cell immediately after cryosection, incubation with spin trap and after covering with Parafilm. Panel B shows the EPRI image obtained after reconstruction and panel $\mathrm{C}$ shows the areas with highest EPRI intensity fused onto the photograph. Panel D shows the immunohistochemistry of an adjacent slice of which the slice where the EPRI was carried out.

Figure 8 shows the areas with highest EPRI intensity fused onto the photographs of the remaining four sections. Plaque 1, section 2 (Panel A), plaque 3, section 1 (Panel B), plaque 2, section 2 (Panel C) and plaque 3, section 2 (Panel D).

The EPR signal intensity for each slice in the EPRI experiment was comparable with the EPR signal intensity for the EPRI of the phantom containing $1 \mathrm{mM}$ TEMPO. The projection (max values) from the final reconstructed EPR images along $\mathrm{z}$ axis (along $B_{0}$ ) for the gelatine/TEMPO phantom (Figure 2 ) and the two sections from two different atherosclerotic plaques shown in Figure 6 (plaque 1, section 1) and Figure 7 (plaque 2, section 1) is shown in Figure 9. The maximum signal intensities were well above noise level.

\section{Histopathology}

The histopathological sections showed ruptured caps in all three patients. Due to technical limitations when cryosectioning heavily calcified tissue, one case was only possible to judge partially. In the six different investigated sections (two separated sections from each of the three patients) the histopathological findings were similar: each cap was partially thin, there was extensive calcification and there was moderately increased amount of foamy macrophages in the cap with extensive macrophage accumulation focally. When comparing the areas of high signal intensity in the EPR images (Figures $6 \mathrm{C}$ and $7 \mathrm{C}$ ) these areas were found within or close to the cap in the area between the lumen and the plaque. These areas showed increased cellularity and signs of activity as shown by frequent accumulations of foamy macrophages (Figure 6 D and 7 D). However, not all foamymacrophage accumulations showed strong signal in the EPR image.

\section{DISCUSSION}

We have developed a method for $2 \mathrm{D}$ visualisation of oxidative stress in biopsies. The developed method was demonstrated in six $(\mathrm{N}=6)$ sections from three different ex vivo carotid atherosclerotic plaque using EPRI combined with the cyclic hydroxylamine spin probe CMH. The EPR spectrum of the oxidised spin probe $\mathrm{CMH}$ is very similar to that of TEMPO and do not depend on the nature of the 
reacted ROS (22). Therefore a phantom of TEMPO with known spatial characteristics and concentration $(1 \mathrm{mM})$ could be used in order to show that the proposed imaging protocol and method for reconstruction gave a reliable result useful for comparison with histopathology. The gelatine/TEMPO phantom used to calibrate and evaluate the developed imaging protocol was not perfectly cylindrical (as shown in Figure 2, Panel A) but visual inspection of the reconstructed EPR image (Figure 2, Panel C) revealed that the there was a good agreement between the distribution of free radicals and the shape and size of the phantom. However, as can been seen in Figure 2, Panel C and in Figure 4, the resolution in the presented method is limited as a consequence of e.g. used gradient strength and the compromise between resolution and signal-to-noise ratio in the Gaussian filter for deconvolution and the Shepp-Logan filter used for the filtered back-projection.

The three plaques in this study were collected from patients with neurological symptoms (symptomatic plaques) with ultrasound characteristics of high vulnerability including ulcerated plaque surfaces. Histology showed local thrombus formation $(\mathrm{N}=1)$ as well as increased macrophage infiltration in the cap $(\mathrm{N}=6)$ that might be associated with high oxidative stress as visualised by EPRI.

While various techniques such as ultrasound, magnetic resonance imaging (MRI), dual energy CT (DECT) and positron emission tomography (PET) are available for visualization of carotid plaques, it is still a clinical challenge to predict the vulnerability for plaque rupture and subsequent embolic stroke. It is well-known that oxidative stress is a key factor in the initiation and progression of atherosclerosis (27) and it has been hypothesized that oxidative stress destabilise the cap of atherosclerotic plaques with subsequent risk of cap rupture (28). The further development of visualisation techniques for imaging of oxidative stress is therefore of clinical interest, especially in the cap whereas high oxidative stress increases the risk for cap rupture.

EPR can also be used to quantify Fe(III) in ex vivo atherosclerotic plaques in addition to studies of oxidative stress. We recently performed an EPR study to assess data concerning differences in iron content as a sign of haemorrhage in clinical silent plaques as compared to plaques provoking neurological symptoms (33). We showed that the Fe(III) distribution varies substantially within atherosclerotic plaques and that plaques from symptomatic patients had significantly higher concentrations of $\mathrm{Fe}(\mathrm{III})$, as well as signs of cap rupture and increased cap macrophage activity (33). Although the method for tissue preparation differed, the previous iron quantification study was based on paraffin embedded and formalin fixed tissue, an increase in cap foamy macrophages was also seen in the currently investigated plaque based on fresh, rapidly frozen sections. 
The histopathological examination revealed that the symptomatic plaques in this study had high presence of foamy macrophages in the caps and signs of ulcerated surface and thrombus formation. Areas with high EPRI intensity were found within or close to the cap in the area between the lumen and the plaque when comparing the areas of high signal in the image obtained using EPRI and histopathology. These areas showed increased cellularity and signs of activity as shown by frequent accumulations of foamy macrophages. However, not all foamy-macrophage accumulations showed strong signal in the EPR image. This could be caused by the technical limitation of using different sections for different analysis, although being close and similar, the sections are not identical. Another interpretation is that there are additional factors, not evident by routine histological evaluation that is necessary for the accumulation of EPRI signal intensity. However, only H\&E staining was performed in this study since the focus was to develop a new method and an imaging protocol for X-Band EPR imaging in ex vivo samples.

It is anticipated that further additional histological evaluation with e.g. immunohistochemistry could yield additional information to evaluate vessel status and related potential factors. These could include iron contents (33) and other factors involved in oxidative stress. Many methods to quantify activity of oxidative stress are done on homogenized tissue, e.g. Western blot and ELISA which hampers the possibility to correlate results from these methods to the EPRI signal intensity pattern. However, it is partially possible to study factors involved in oxidative stress in histological sections using immunohistochemistry with antibodies against e.g. superoxide dismutase and active caspase-3. With the potential of the EPRI method shown in the current study this opens up for future studies correlating the EPRI signal pattern to tissue and cellular factors in atherosclerotic plaques.

\section{CONCLUSIONS}

We have demonstrated that the distribution of oxidative stress in a biopsy of ex vivo atherosclerotic plaques can be mapped using EPRI of a ROS sensitive spin-probe. The method is based on incubation with the spin probe $\mathrm{CMH}$ to observe signal accumulation in regions with possible high oxidative stress and to develop a Matlab code for signal deconvolution and image reconstruction. The methodology might give new and meaningful comparison between histopathology and redoxcondition in various biopsies. Thus, further work, including a thorough and systematic evaluation on the performance of the proposed technique on different types of biopsies in comparisons with other optical imaging techniques, must be performed to further validate the proposed technique.

\section{ACKNOWLEDGMENTS}


H. Gustafsson acknowledges The Swedish Research Council (diarienr 2009-5430) for financial support. M. Engström acknowledges the Research Council of Southeast Sweden (FORSS) for financial support. M. Lindgren thanks Linköping University for a visiting professor scholarship. Sandeep Koppal is acknowledged for sample handling and transport. Per Hammarström, Sofie Nyström and Daniel Sjölander are acknowledged for valuable discussions.

\section{REFERENCES}

1. Winterbourn CC. Reconciling the chemistry and biology of reactive oxygen species. Nat Chem Biol 2008; 4: 278-286.

2. Dickinson BC, Chang CJ. Chemistry and biology of reactive oxygen species in signaling or stress responses. Nat Chem Biol 2011; 7: 504-511.

3. Valko M, Leibfritz D, Moncola J, Cronin MTD, Mazur M, Telser J. Free radicals and antioxidants in normal physiological functions and human disease. Int J Biochem Cell Biol 2007; 39: 44-84.

4. Drummond GR, Selemidis S, Griendling KK, Sobey CG. Combating oxidative stress in vascular disease: NADPH oxidases as therapeutic targets. Nat Rev Drug Discov 2011; 10: 453-471.

5. Cai H, Harrison DG. Endothelial dysfunction in cardiovascular diseases: The role of oxidant stress. Circ Res 2000; 87: 840-844.

6. Madamanchi NR, Vendrov A, Runge MS. Oxidative Stress and Vascular Disease. Arterioscler Thromb Vasc Biol 2005; 25: 29-38.

7. Eaton GR, Eaton SS, Ohno K. EPR imaging and in vivo EPR. Boca Raton FL: CRC Press. 1991.

8. Bobko AA, Eubank TD, Voorhees JL, Efimova OV, Kirilyuk IA, Petryakov S, Trofimiov DG, Marsh CB, Zweier JL, Grigorev IA, Samouilov A, Khramtsov VV. In Vivo Monitoring of pH, Redox Status, and Glutathione Using L-Band EPR for Assessment of Therapeutic Effectiveness in Solid Tumors. Magn Reson Med 2012; 67: 1827-1836.

9. Elas M, Ichikawa K, Halpern HJ. Oxidative Stress Imaging in Live Animals with Techniques Based on Electron Paramagnetic Resonance. Radiat Res 2012; 177: 514-523. 
10. Ji J, Kline AE, Amoscato A, Samhan-Arias AK, Sparvero LJ, Tyurin VA, Tyurina YY, Fink B, Manole MD, Puccio AM, Okonkwo DO, Cheng JP, Alexander H, Clark RSB, Kochanek PM, Wipf P, Kagan VE, Bayır H. Lipidomics identifies cardiolipin oxidation as a mitochondrial target for redox therapy of brain injury. Nat Neurosci 2012; 15: 1407-1415.

11. Swartz HM, Liu KJ, Goda F, Walczak T. India Ink: A Potential Clinically Applicable EPR Oximetry Probe. Magn Reson Med 1994; 31: 229-232.

12. Goda F, Liu KJ, Walczak T, O'Hara JA, Jiang J, Swartz HM. In vivo Oximetry Using EPR and India Ink. Magn Reson Med 1995; 33: 237-245.

13. Eaton SS, Eaton GR. The world as viewed by and with unpaired electrons. J Magn Reson 2012; 223: 151-163.

14. Berliner LJ. In vivo EPR (ESR): theory \& applications. New York: Kluwer Academic/Plenum Publishers. 2003; 99-152 p.

15. Kruczala K, Motyakin MV, Schlick S. 1D and 2D Electron Spin Resonance Imaging (ESRI) of Nitroxide Radicals in Stabilized Poly(acrylonitrile-butadiene-styrene) (ABS): UV vs Thermal Degradation. J. Phys. Chem. B, 2000; 104: 3387-3392.

16. Halliwell B, Gutteridge JMC. Free Radicals in Biology and Medicine. New York: Oxford University Press Inc. 2007; 268 - 340 p.

17. Finkelstein E, Rosen GM, Rauckman EJ. Spin Trapping. Kinetics of the reaction of superoxide and Hydroxyl radicals with nitrones. J Am Chem Soc 1980; 102: 4994 - 4999.

18. Herrling T, Fuchs J, Rehberg J, Groth N. UV-induced free radicals in the skin detected by ESR spectroscopy and imaging using nitroxides. Free Radic Biol Med 2003; 35: 59-67.

19. Kuppusamy P, Li H, Ilangovan G, Cardounel AJ, Zweier JL, Yamada K, Krishna MC, Mitchell JB. Noninvasive Imaging of Tumor Redox Status and Its Modification by Tissue Glutathione Levels. Cancer Res 2002; 62: 307-312. 
20. Dikalov S, Skatchkov M, Bassenge E. Quantification of Peroxynitrite, Superoxide, and Peroxyl Radicals by a New Spin Trap Hydroxylamine 1-Hydroxy-2,2,6,6-tetramethyl-4-oxo-piperidine. Biochem Biophys Res Commun 1997; 230: 54-57.

21. Dikalov SI, Li W, Mehranpour P, Wang SS, Zafari AM. Production of extracellular superoxide by human lymphoblast cell lines: Comparison of electron spin resonance techniques and cytochrome $\mathrm{C}$ reduction assay. Biochem Pharmacol 2007; 73: 972 - 980.

22. Dikalov S, Griendling KK, Harrison DG. Measurement of Reactive Oxygen Species in Cardiovascular Studies. Hypertension 2007; 49: 717-727.

23. Dikalov SI, Kirilyuk IA, Voinov M, Grigor'ev IA. EPR detection of cellular and mitochondrial superoxide using cyclic hydroxylamines. Free Radic Res 2001; 45: 417-430.

24. Kuzkaya N, Weissmann N, Harrison DG, Dikalov S. Interactions of peroxynitrite with uric acid in the presence of ascorbate and thiols: Implications for uncoupling endothelial nitric oxide synthase. 2005; 70: 343-354.

25. Dikalov SI, Dikalova AE, Mason RP. Noninvasive diagnostic tool for inflammation-induced oxidative stress using electron spin resonance spectroscopy and an extracellular cyclic hydroxylamine. Arch Biochem Biophys. 2002; 402: 218-226.

26. Dikalova A, Clempus R, Lassegue B, et al. Nox1 overexpression potentiates angiotensin IIinduced hypertension and vascular smooth muscle hypertrophy in transgenic mice. Circulation 2005; 112: 2668-2676.

27. Stocker R, Keaney Jr JF. Role of Oxidative Modifications in Atherosclerosis. Physiol Rev 2004; 84: $1381-1478$.

28. Rajagopalan S, Meng XP, Ramasamy S, Harrison DG, Galis ZS. Reactive oxygen species produced by macrophage-derived foam cells regulate the activity of vascular matrix metalloproteinases in vitro. Implications for atherosclerotic plaque stability. J Clin Invest 1996; 98: 2572-2579. 
29. Jogestrand T, Eiken O, Nowak J. Relation between the elastic properties and intima-media thickness of the common carotid artery. Clin Physiol Funct Imaging 2003; 223: 134-137.

30. Stoll S, Schweiger A. EasySpin, a comprehensive software package for spectral simulation and analysis in EPR. J Magn Reson 2006; 178: 42-55.

31. Hornak JP, Moscicki JK, Schneider DJ, Freed JH. Diffusion coefficients in anisotropic fluids by ESR imaging of concentration profiles. J Chem Phys 1986; 84: 3387 - 3395.

32. Barnett HJ, Taylor DW, Eliasziw M, Fox AJ, Ferguson GG, Haynes RB, Rankin RN, Clagett GP, Hachinski VC, Sackett DL, Thorpe KE, Meldrum HE, Spence JD. Benefit of carotid endarterectomy in patients with symptomatic moderate or severe stenosis: North American Symptomatic Carotid Endarterectomy Trial Collaborators. N Engl J Med 1998; 339: 1415-1425.

33. Gustafsson H, Hallbeck M, Norell M, Lindgren M, Engström M, Rosén A, Zachrisson H. Fe(III) distribution varies substantially within and between atherosclerotic plaques Magn Reson Med 2014; 71: 885-892. 


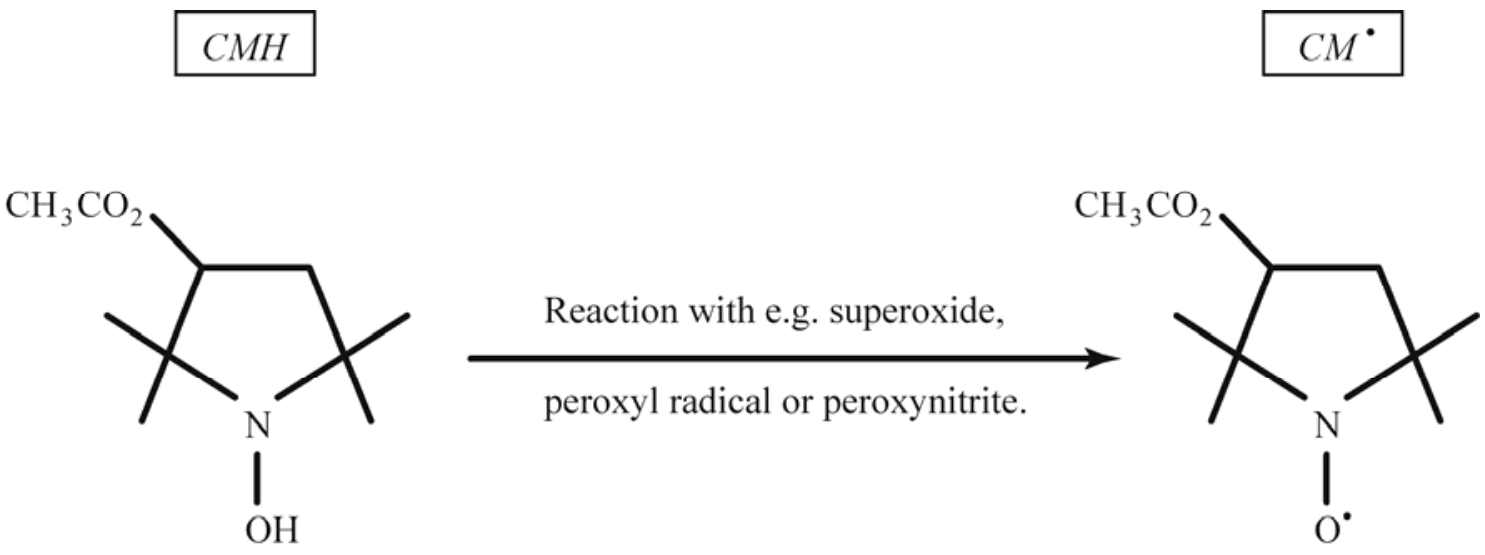

Figure 1. The oxidation of the diamagnetic spin probe 1-hydroxy-3-methoxycarbonyl-2,2,5,5tetramethylpyrrolidine $(\mathrm{CMH})$ leads to the formation of the paramagnetic 3-methoxycarbonyl-proxyl nitroxide $(\mathrm{CM} \bullet)$.

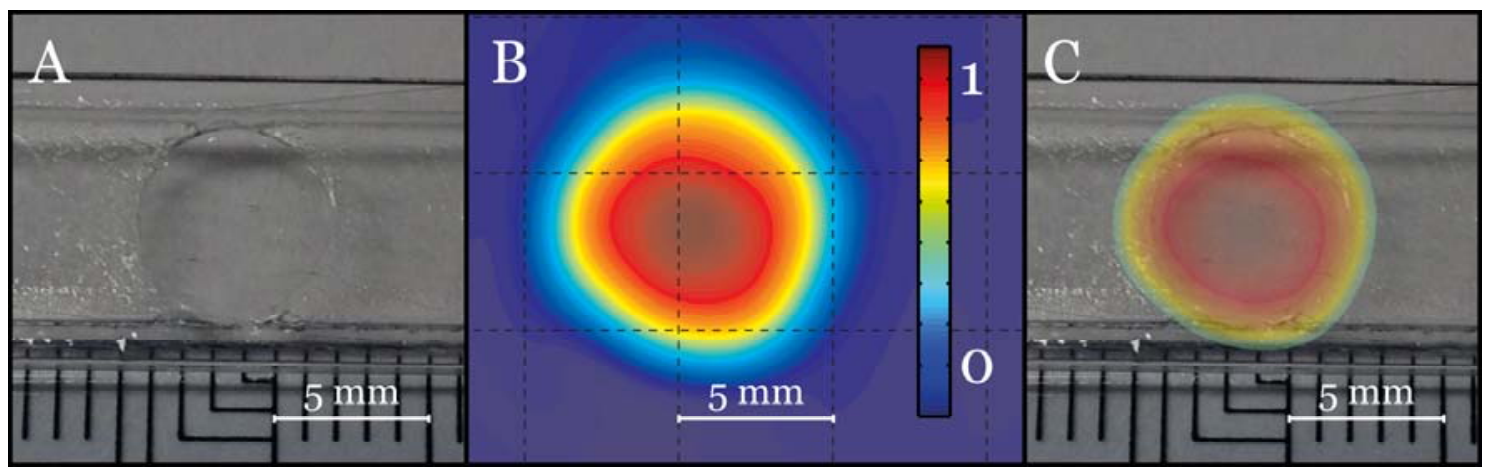

Figure 2. Photograph of the gelatine/TEMPO phantom (Panel A). EPRI of the phantom (Panel B).

Signal intensity was colour-coded in arbitrary EPRI units as indicated by the colour bar (yellow to red indicates high EPRI signal intensity and green to blue indicates low to no EPRI signal intensity). Semi-transparent EPRI-image overlaid on the photograph (Panel C). 

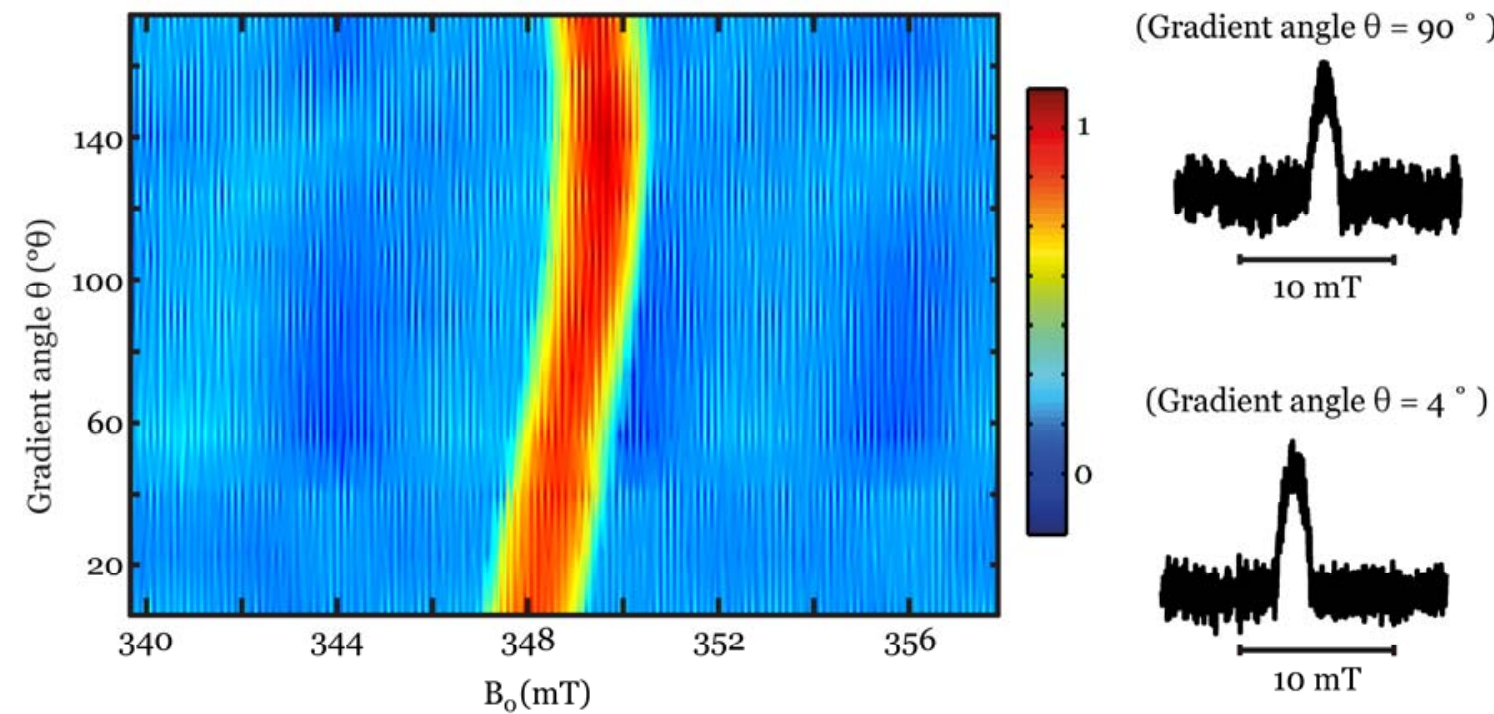

Figure 3. 2D plot of the EPR spectra after deconvolution as a function of gradient angle in the ZY plane (to the left in the figure). Signal intensity was colour-coded in arbitrary EPR units as indicated by the colour bar (yellow to red has more positive amplitude and green to blue is indicative of less amplitude). EPR spectra for two particular gradient angles $\left(\theta=4^{\circ}\right.$ and $\left.\theta=90^{\circ}\right)$ (right).
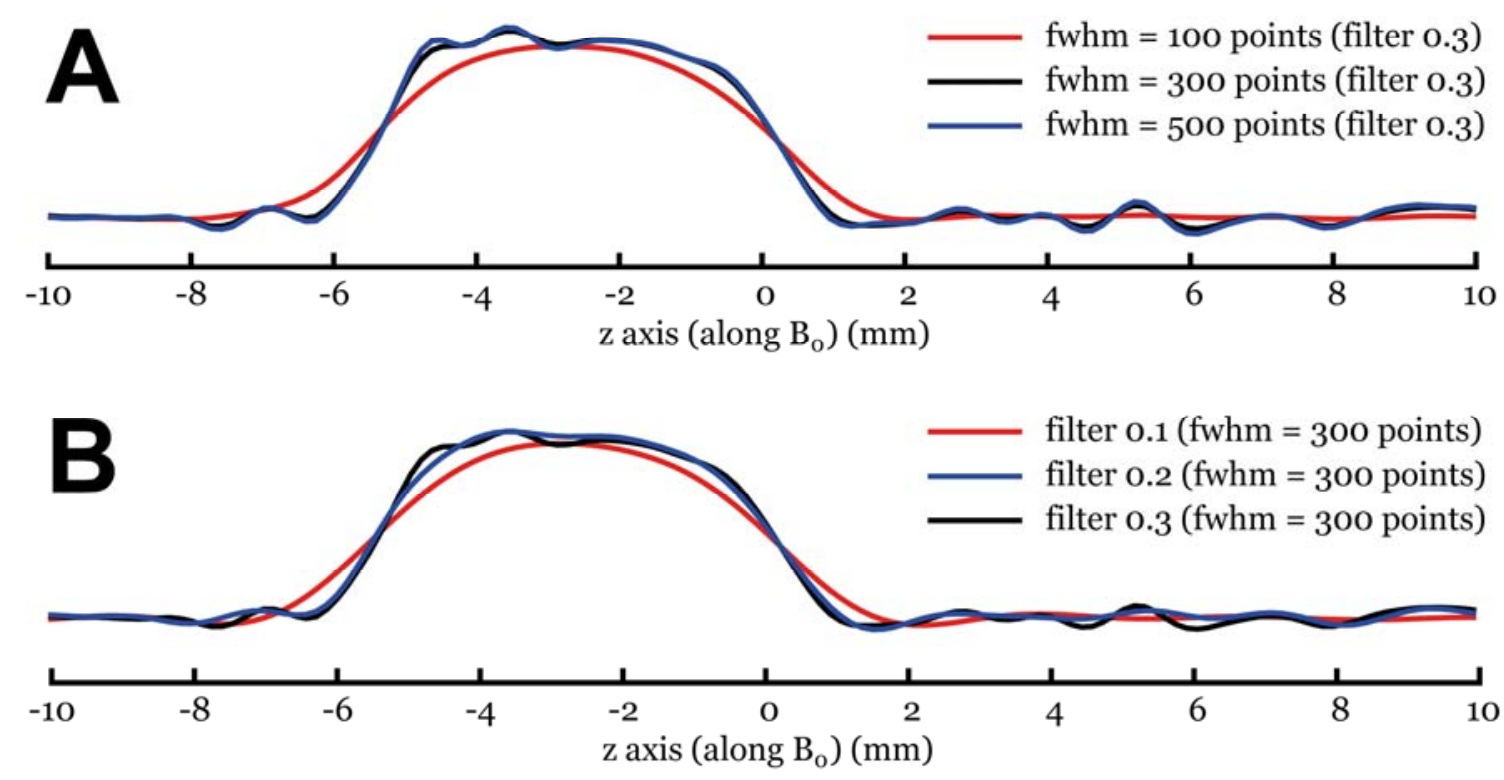

Figure 4. EPRI signal intensity along on line in the EPR image as a function of the $\mathrm{z}$-axis (along $\mathrm{B}_{0}$ ) of the $1 \mathrm{mM}$ TEMPO /gelatine phantom shown in Figure 2A. Panel A shows EPRI signal intensity for 
three different Gaussian filters for spectral deconvolution. Panel B shows EPRI signal intensity for three different Shepp-Logan filters used in the filtered back-projection.

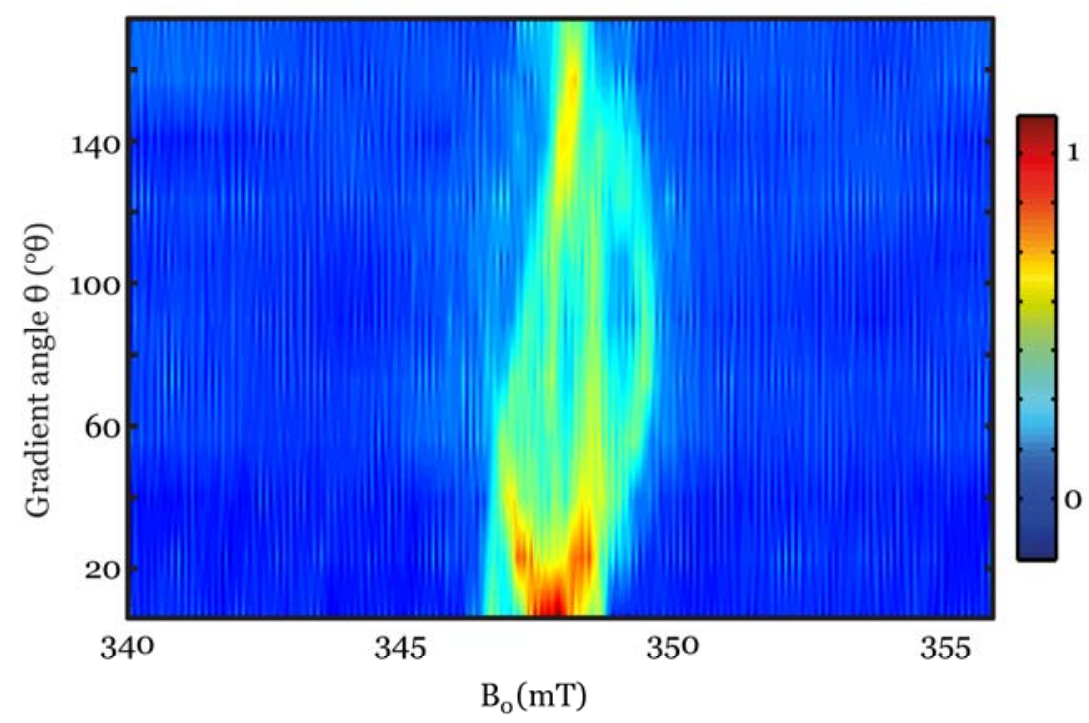

(Gradient angle $\theta=90^{\circ}$ )

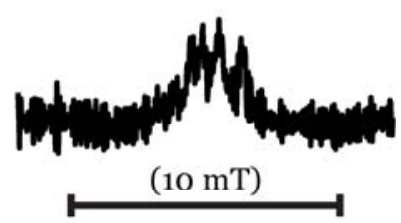

(Gradient angle $\theta=4^{\circ}$ )

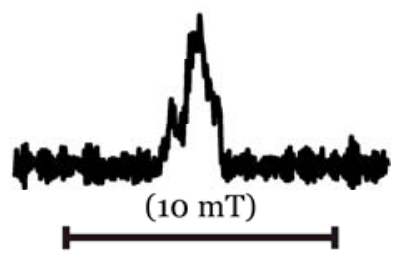

Figure 5. EPR spectra after deconvolution for one section of one of the studied atherosclerotic plaques (plaque 1, section 1) (left). EPR spectra for two particular gradient angles $\left(\theta=4^{\circ}\right.$ and $\left.\theta=90^{\circ}\right)$ are shown to the right. 

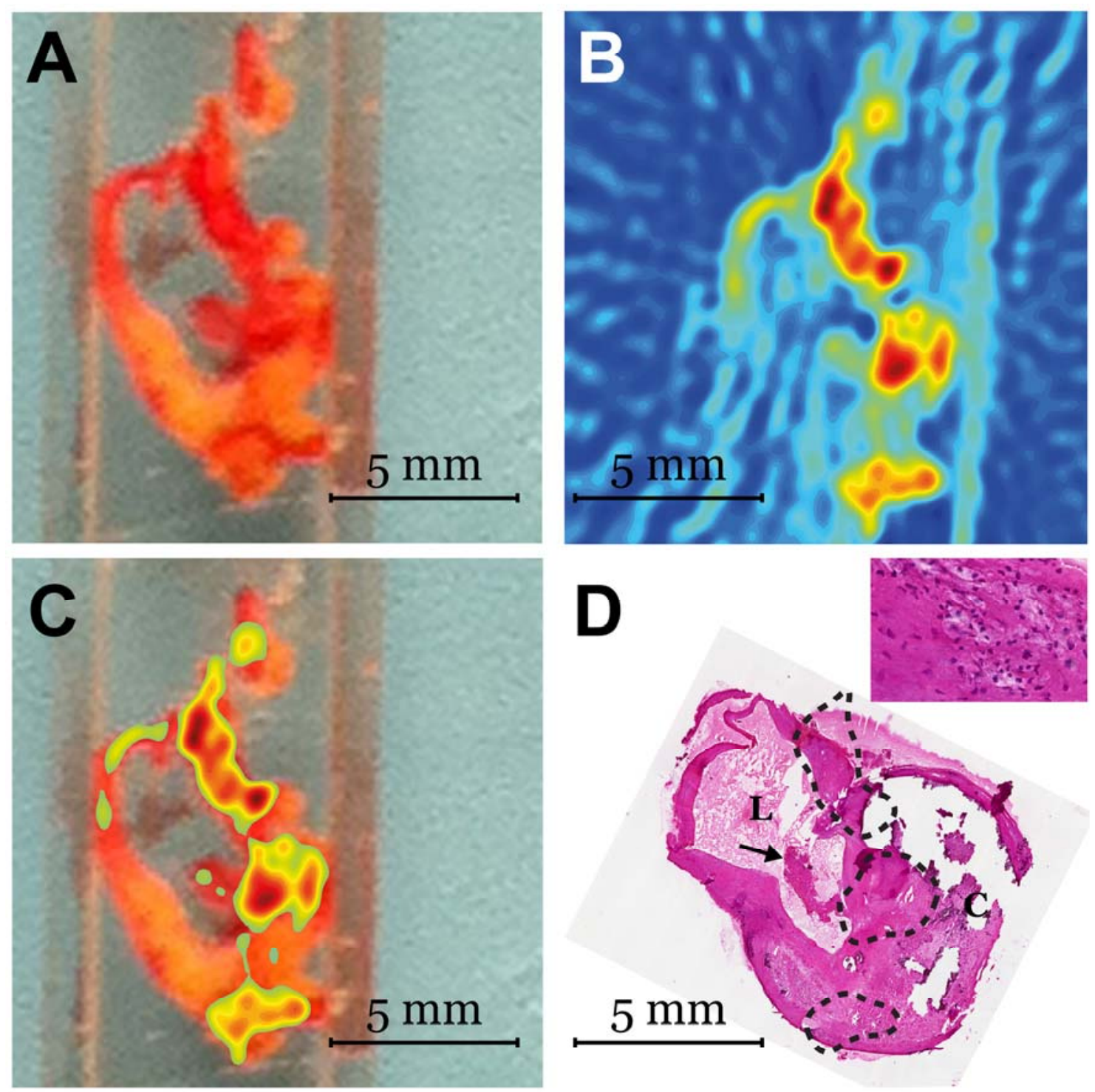

Figure 6. Photograph of the section (plaque 1, section 1) used to obtain the data in shown in Figure 5 located on the EPR tissue cell immediately after cryosection, incubation with spin trap and after covering with Parafilm (panel A). Panel B: EPR image obtained after reconstruction of the data shown in figure 5 . Note that most part of the plaque gives some EPRI intensity but certain areas shows high concentration of spin probe signal. Panel C shows the areas with highest EPRI intensity fused onto the photograph. Panel D shows the histology of an adjacent slice with the high signalling areas superimposed as dashed outlines. The arrow points at the thrombus formation protruding into the lumen from the wall. L; lumen, C; lipid core. Scale bar correspond to $5 \mathrm{~mm}$. The high magnification insert shows aggregations of foamy macrophages taken from the upper high signalling area. 

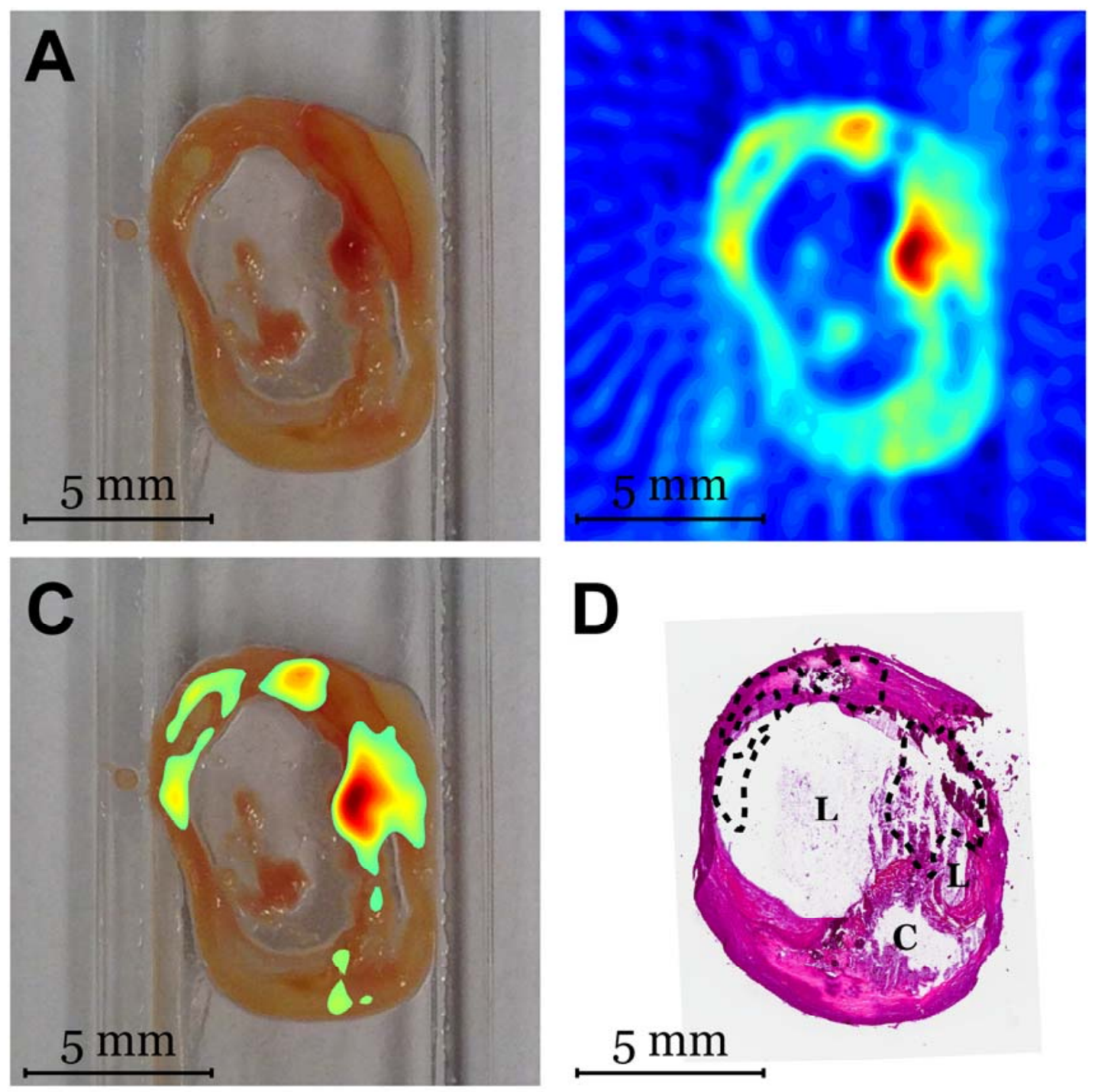

Figure 7. EPRI similar to Figure 6 but obtained from a different section from a different atherosclerotic plaque (plaque 2, section 1). Panel A shows a photograph of the section located on the EPR tissue cell immediately after cryosection, incubation with spin trap and after covering with Parafilm. Panel B shows the EPRI image obtained after reconstruction and panel C shows the areas with highest EPRI intensity fused onto the photograph. Panel D shows the immunohistochemistry of an adjacent slice of which the slice where the EPRI was carried out. 

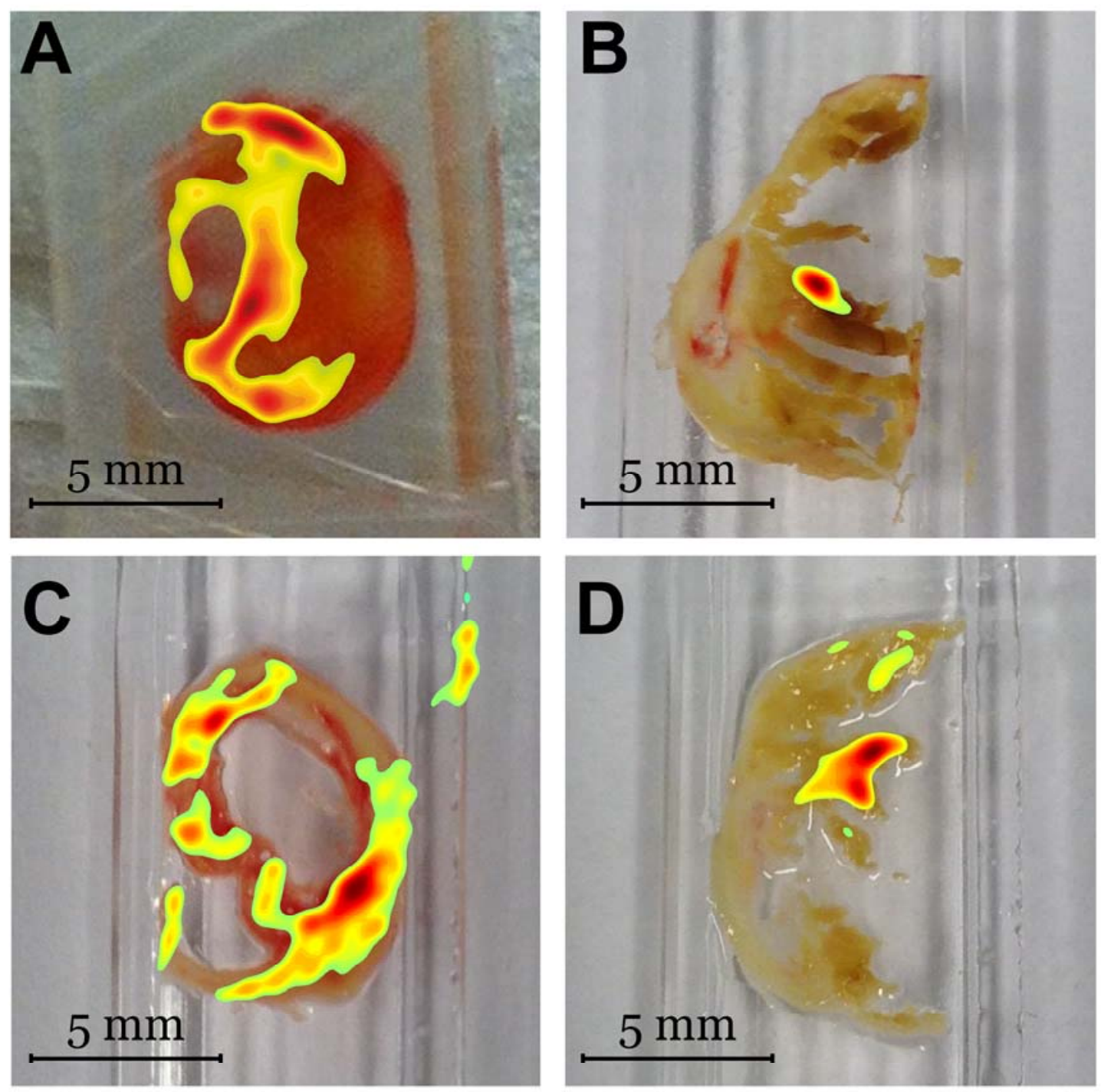

Figure 8. The areas with highest EPRI intensity fused onto the photographs of the remaining four sections. Plaque 1, section 2 (Panel A), plaque 3, section 1 (Panel B), plaque 2, section 2 (Panel C) and plaque 3, section 2 (Panel D). 


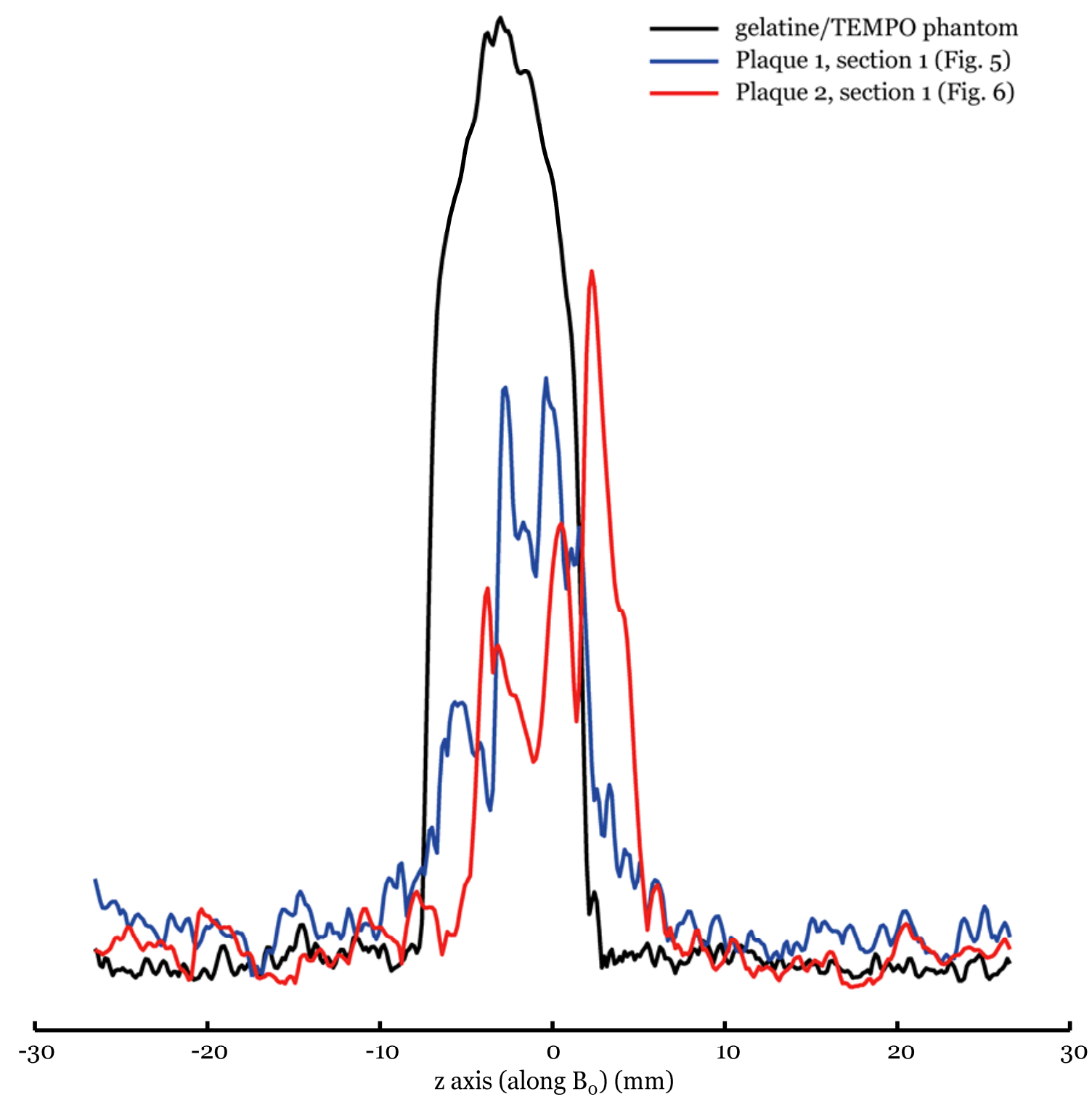

Figure 9. The figure shows the projections (max values) along $\mathrm{z}$ axis (along $\mathrm{B}_{0}$ ) from the final reconstructed EPR images shown in Figure 2 for the gelatine/TEMPO phantom, and from the two sections from two different atherosclerotic plaques shown in Figure 6 (plaque 1, section 1) and Figure 7 (plaque 2, section 1). 


\section{SUPPORTING INFORMATION}

Matlab m-file for import of Bruker raw-data, deconvolution, back projection and image reconstruction. 
1 function [] = EPRI_tissue_cell

$2 \%$ File which reconstructs 2D EPRI from X Band EPR.

$3 \%$

$4 \%$ function [] = EPRI_tissue_cell

$5 \%$

6 \% Håkan Gustafsson, December 2012.

7

8 clear all

9 close all

10

$11 \%$ Import of files.

12 file_name_1 = (input('Filename for measurement with gradient: ','s'));

$13[\mathrm{q} 1 \mathrm{a}, \mathrm{q} 1 \mathrm{~b}, \mathrm{q} 1 \mathrm{c}]=$ eprload(file_name_1);

14 file_name_2 =(input('Filename for measurement without gradient: ','s'));

$15[\sim, w 2]=$ eprload(file_name_2);

16

$17 \quad \mathrm{r} 1(:, 1)=\mathrm{q} 1 \mathrm{a}\{1,1\} . / 10 ; \%$ Magnetic field.

$18 \mathrm{r} 2(:, 1)=\mathrm{q} 1 \mathrm{a}\{1,2\} ; \%$ Gradient angles.

$19 \mathrm{r} 3=\operatorname{rot} 90(\mathrm{q} 1 \mathrm{~b})$;

20

$21 \%$ Base line subtraction.

$22[$ raws $\sim]=\operatorname{size}(\mathrm{r} 3)$;

23 for $\mathrm{r} 4=1$ :raws

$24 \mathrm{k}=(\mathrm{r} 3(\mathrm{r} 4, \mathrm{end})-\mathrm{r} 3(\mathrm{r} 4,1)) /(\mathrm{r} 1(\mathrm{end}, 1)-\mathrm{r} 1(1,1)) ; \% \mathrm{y}=\mathrm{kx}+\mathrm{m}$

$25 \mathrm{~m}=\mathrm{r} 3(\mathrm{r} 4,1)-\mathrm{r} 1(1,1) * \mathrm{k}$;

$26 \quad$ straight $(\mathrm{r} 4,:)=\mathrm{k} * \mathrm{r} 1(:, 1)+\mathrm{m}$;

27 end

28 r5=r3-straight;

29

30 figure,

$31 \operatorname{subplot}\left(2,2,\left[\begin{array}{ll}1 & 2\end{array}\right]\right)$, contourf(r1(1:2:end),r2(1:2:end),r5(1:2:end,1:2:end), 100,'LineStyle','none')

$32 \mathrm{c}=$ colorbar;

33 ylabel(c,'EPR signal intensity (arbitrary units)');

34 colormap jet

35 xlabel('B_0 (mT)');

36 ylabel('Gradient angle $\backslash$ theta $\left({ }^{\wedge}\{0\} \backslash\right.$ theta $\left.) '\right)$; 


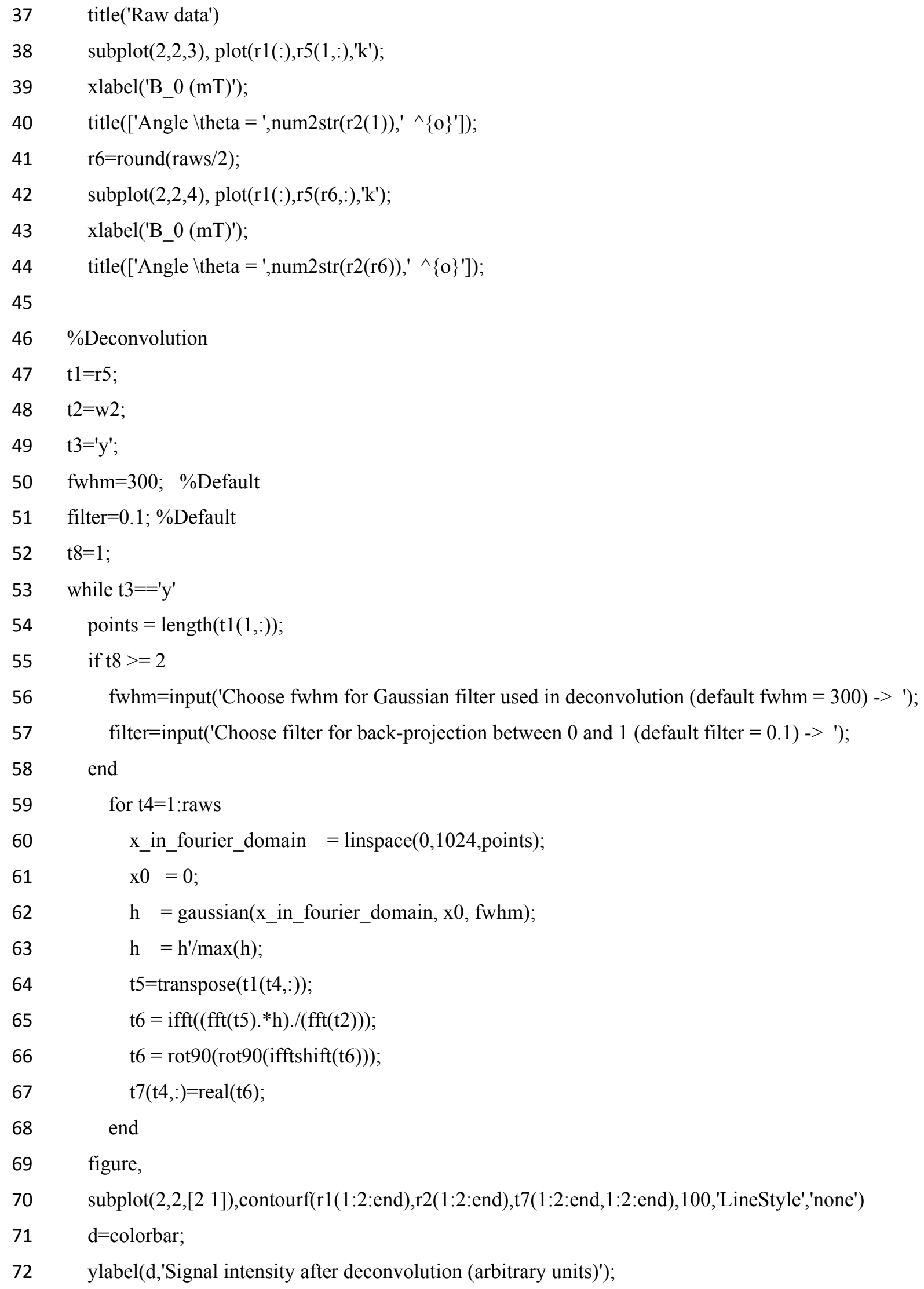




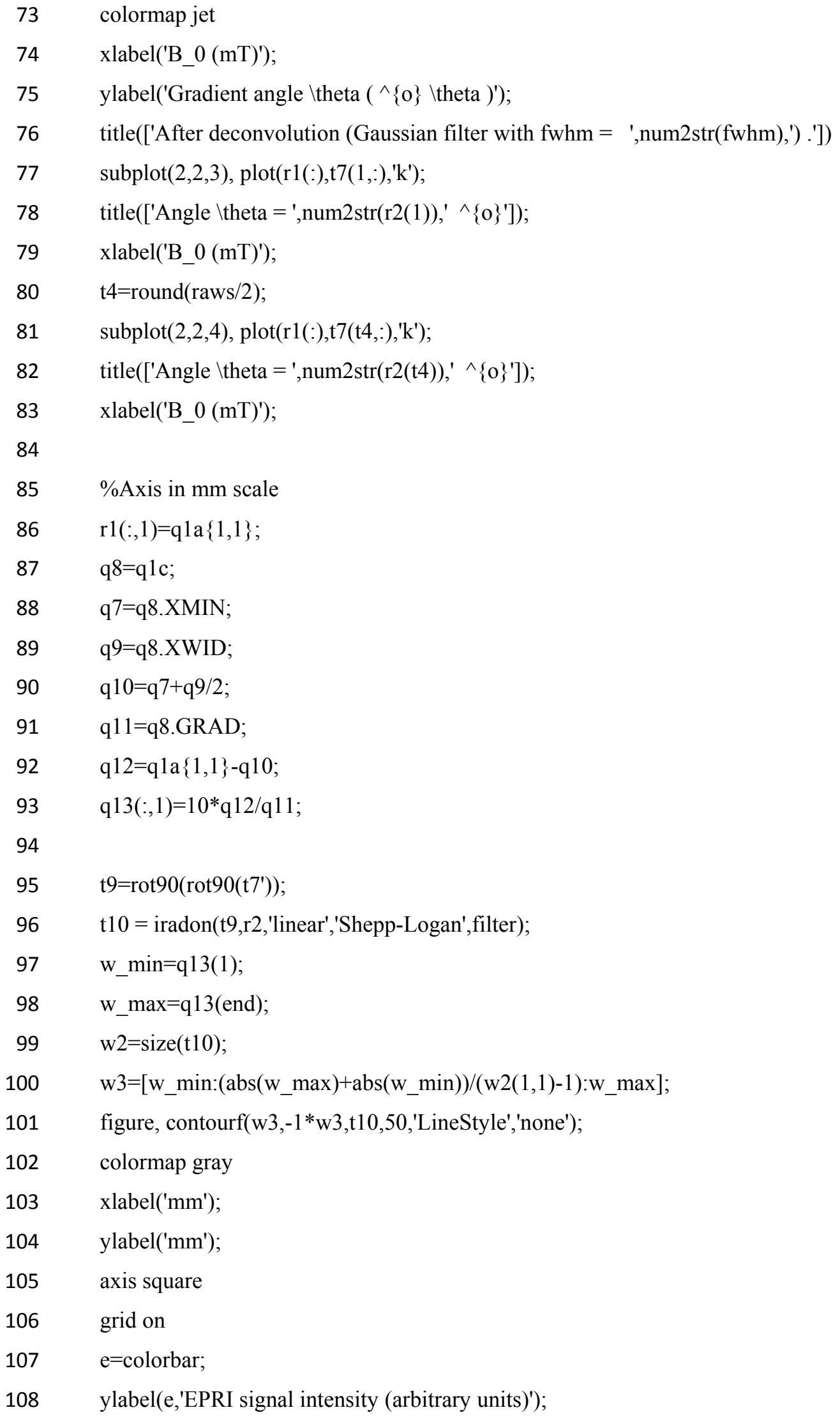




$$
\text { colormap jet }
$$

110 title(['EPRI of sample ',num2str(file_name_1),' after deconvolution (Gaussian filter fwhm =

111 ',num2str(fwhm), ') and filtered backprojection (Shepp-Logan filter = ',num2str(filter), ').'])

$112 \mathrm{t} 3=$ input('Try other settings for deconvolution filter and/or filter for backprojection? Yes (y) or no

113 (n)? ','s');

$114 \quad \mathrm{t} 8=\mathrm{t} 8+1$;

115 end

116

117 assignin('base','signal',t10);

118 disp('To plot image write e.g. imagesc(x_axis,y_axis,signal).')

119

120

\%Projection along each axis.

121

122

for $\mathrm{y} 1=1: \mathrm{w} 2$

123

$$
\mathrm{y} 2 \max (\mathrm{y} 1)=\max (\mathrm{t} 10(\mathrm{y} 1,1: \mathrm{end}))
$$

124 end

125

126

for $\mathrm{y} 1=1: \mathrm{w} 2$

127

$$
\mathrm{y} 3 \max (\mathrm{y} 1)=\max (\mathrm{t} 10(1: \mathrm{end}, \mathrm{y} 1))
$$

128

end

129

130

figure,plot(w3,y2max,'k');

131 grid on

132 title('Projection (max values) along y axis (sample tube axis).')

133 axis square

134 xlabel('y axis (mm) (along sample tube)');

135 ylabel('EPR signal intensity (arbitrary units)');

136 legend('max value along axis')

137 figure,plot(w3,y3max,'k');

138 grid on

139 title('Projection (max values) along $\mathrm{z}$ axis (sample B_0).')

140 axis square

141 xlabel('z axis (mm) (along B_0)');

142 ylabel('EPR signal intensity (arbitrary units)');

143 legend('max value along axis')

144 assignin('base','projection_along_y_axis',y2max); 
145 assignin('base','projection_along_z_axis',y3max);

146 assignin('base','x_axis',w3);

147 assignin('base','y_axis',w3); 